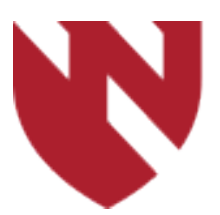

October 2021

\title{
The Isolated Fibular Fracture: Successful Outcomes with Non- operative, Immediate Weight Bearing Despite Stress Positive Radiographs
}

\author{
Phillip Thomas \\ University of Nebraska Medical Center \\ Leonid Grossman \\ University of Nebraska Medical Center \\ Sara Putnam \\ University of Nebraska Medical Center \\ Justin C. Siebler \\ University of Nebraska Medical Center \\ Elizabeth Lyden \\ Panorama Orthopedics and Spine Center
}

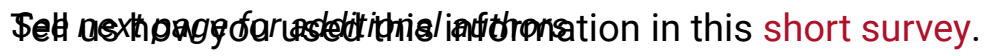

Follow this and additional works at: https://digitalcommons.unmc.edu/gmerj

Part of the Higher Education Commons, and the Medicine and Health Sciences Commons

\section{Recommended Citation}

Thomas, P., Grossman, L., Putnam, S., Siebler, J. C., Lyden, E., , Mormino, M. The Isolated Fibular Fracture: Successful Outcomes with Non-operative, Immediate Weight Bearing Despite Stress Positive Radiographs. Graduate Medical Education Research Journal. 2021 Oct 04; 3(1).

https://digitalcommons.unmc.edu/gmerj/vol3/iss $1 / 20$

This Conference Proceeding is brought to you for free and open access by DigitalCommons@UNMC. It has been accepted for inclusion in Graduate Medical Education Research Journal by an authorized editor of DigitalCommons@UNMC.For more information, please contact digitalcommons@unmc.edu. 
The Isolated Fibular Fracture: Successful Outcomes with Non-operative, Immediate Weight Bearing Despite Stress Positive Radiographs

\section{Creative Commons License}

\section{(c) (i) (9)}

This work is licensed under a Creative Commons Attribution-Noncommercial-No Derivative Works 4.0 License.

Authors

Phillip Thomas, Leonid Grossman, Sara Putnam, Justin C. Siebler, Elizabeth Lyden, and Matthew Mormino 


\section{Newsworthiness of Neurological Research}

Isha Snehal' ${ }^{1}$, Appaji Rayi ${ }^{2}$, Vineet Punia ${ }^{3}$

${ }^{1}$ University of Nebraska Medical Center, College of Medicine, Department of Neurological Sciences ${ }^{2}$ Ohio State Wexner Medical Center, Department of Neurology

${ }^{3}$ Epilepsy Center, Cleveland Clinic Foundation, Ohio

Mentor: Vineet Punia

Program: Neurology

Type: Original Research

Background: Informing the public of the latest scientific breakthroughs is an important result of research. Popular news media remains an impactful means of research dissemination. We, therefore, evaluated the factors that determine the newsworthiness of neurological research. Our objective was to evaluate news coverage received by neurological research and factors associated with its newsworthiness.

Methods: Original research articles from the year 2016 in the top 5 neurological journals, based on impact factor, were extracted. Individual research articles were reviewed for the sub-specialty, study design, funding source, continent and institute of origin. Dimensions, an online platform, was used to find the number of news and Twitter mentions and open access status. Articles receiving a news mention were considered 'newsworthy.' Logistic regression models for "newsworthiness" were performed.

Results: Of the 1050 research articles included, 551 (52.5\%) were newsworthy, with a median of 2 [inter-quartile range $(\mathrm{IQR})=1-10$ ] news mentions. Newsworthy articles were significantly more likely to have a higher number of tweets [Odds ratio $(\mathrm{OR})=1.04(1.03-1.05), \mathrm{p}<0.001]$ and open-access $(\mathrm{OR}=1.63(1.22-2.17), \mathrm{p}=$ $0.02)$. Newsworthiness was not associated with study design, article type, funding status, continent, or institute of origin. Publication in JAMA Neurology [OR $=2.86(1.39-$ $6.1), \mathrm{p}=0.005$ ] and the Lancet Neurology $[\mathrm{OR}=2.86(1.39-6.1), \mathrm{p}=0.005]$ affected newsworthiness. Articles on cognitive neurology were 22 times [OR $=22.62$ $(11.48-47.95), \mathrm{p}<0.001]$ more likely to be newsworthy.

Conclusion: Almost half of the research articles published in top neurology journals do not receive any news mention. Newsworthiness is associated with the popularity of research articles on social media. Studies on cognitive neurology seem to be of high interest in the news. Future research to understand the impact of newsworthiness on clinical outcomes is required.

https://doi.org/10.32873/unmc.dc.gmerj.3.1.038

\section{Pediatric Orthopaedic Trauma: What Defines "Pediatric?"}

Erin L. Stockwell', Susan A. Scherl ${ }^{1,2}$, Maegen J. Wallace ${ }^{1,2}$, Matthew A. Halanski ${ }^{1,2}$, Brian P. Hasley ${ }^{1,2}$ ${ }^{1}$ University of Nebraska Medical Center, College of Medicine, Department of Orthopaedic Surgery ${ }^{2}$ Children's Hospital and Medical Center, Department of Orthopaedic Surgery

Mentors: Brian Hasley, Susan Scherl

Program: University of Nebraska Medical Center Orthopaedic Surgery Residency Program

Type: Original Research

Background: Pediatric trauma patients have been shown to have better outcomes when treated at pediatric trauma centers, highlighting the importance of understanding the unique physiologic characteristics of pediatric patients. This suggests that pediatric orthopaedic trauma may be best defined physiologically, rather than by age. This study seeks to determine how pediatric orthopaedic trauma patients are defined and managed at leading pediatric orthopaedic departments.
Methods: A 19-question survey was sent to one designated member within the orthopaedic department of each of the Top 50 Children's Hospitals for Orthopaedics (per U.S. News and World Report 2019). Questions utilized multiple choice, yes/no, and open-ended response formats.

Results: Forty eight of the 50 surveys were completed. Pediatric orthopaedic trauma is defined by age at $78 \%$ of institutions. The mean upper-age limit was 18 years (range 14-26 years). All institutions treat simple and complex fractures in skeletally immature patients. Long bone fractures, complex periarticular fractures, and operative pelvic and acetabular fracture in skeletally mature patients were treated by $96 \%, 67 \%$ and $25 \%$ of respondents, respectively.
Conclusion: Nearly $80 \%$ of leading pediatric orthopaedic hospitals define orthopaedic trauma patients based on age. Most pediatric orthopaedists manage complex periarticular and long bone fractures in skeletally mature patients. Pediatric orthopaedist referral rates are increasing, and there is a growing concern for a shortage of pediatric orthopaedists. We challenge the norm of defining pediatric orthopaedic trauma patients based on an arbitrary age cutoff; defining based on physeal closure may improve outcomes while helping offload the burden of patient volume facing pediatric orthopaedists.

https://doi.org/10.32873/unmc.dc.gmerj.3.1.047

\section{The Isolated Fibula Fracture: Successful Outcomes with Non-operative, Immediate Weight Bearing Despite Stress Positive Radiographs Phillip Thomas ${ }^{1}$, Leonid Grossman², Sara Putnam¹, Justin Siebler ${ }^{1}$, Elizabeth Lyden ${ }^{3}$, Matthew Mormino \\ ${ }^{1}$ University of Nebraska Medical Center, College of Medicine, Department of Orthopedic Surgery \\ ${ }^{2}$ University of Nebraska Medical Center, College of Public Health, Department of Biostatistics \\ ${ }^{3}$ Panorama Orthopedics and Spine Center}

Mentor: Matthew Mormino

Program: Orthopedic Surgery

Type: Original Research
Background: Treatment of isolated distal fibula fractures relies on identifying whether a fracture is stable or unstable. Stress radiographs are the current gold standard for evaluating stability in ankle fractures. We present a novel method of evaluation for ankle fracture stability and treatment using an immediate weight-bearing (WB) protocol. 
Methods: Patients with isolated distal fibula fractures were prospectively enrolled between 2018 and 2020. Patients with MCS $<4 \mathrm{~mm}$ on standard NWB ankle radiographs with isolated distal fibula fractures had gravity stress views obtained in the emergency department. Patients were placed in a walking boot and instructed to weight-bear as tolerated (WBAT). WB radiographs were obtained one week later. If the MCS was $>4 \mathrm{~mm}$ on followup WB radiographs, surgical intervention was performed. If MCS was $<4 \mathrm{~mm}$, then management continued with WBAT in a walking boot. AOFAS scores were obtained at three months and six months for all patients.
Results: 44 patients met inclusion criteria for analysis, 22 patients had a MCS $>4 \mathrm{~mm}$ on initial gravity stress views. All 22 of those patients, as well as the remaining 22 patients, continued to show MCS $<4 \mathrm{~mm}$ on all follow-up weight-bearing radiographs up to six-month follow-up while using our novel WBAT protocol. The average AOFAS scores were comparable for both groups.

Conclusion: Instructing patients to WBAT in a walking boot is a viable method to determine stability in isolated distal fibula fractures. Given that all patients at subsequent follow-up had a MCS $<4 \mathrm{~mm}$, including those who widened on initial stress radiographs, an immediate WB protocol may offer an improved indication of ankle stability when compared to the current gold standard stress radiographs. Finally, we saw excellent patientreported outcomes utilizing our immediate weight-bearing protocol.

https://doi.org/10.32873/unmc.dc.gmerj.3.1.035

\author{
Guillain Barre Syndrome as a Complication of COVID-19: A Systematic Review \\ Mohammad Aladawi ${ }^{1}$, Mohamed Elfil', Baha Abu-Esheh², Deaa Abu Jazar ${ }^{3}$, Ahmad Armouti ${ }^{4}$, Ahmed Bayoumi ${ }^{5}$, Ezequiel Piccione ${ }^{1}$ \\ ${ }^{1}$ University of Nebraska Medical Center, College of Medicine, Department of Neurological Sciences \\ ${ }^{2}$ Mercy Hospital, Department of Neurology \\ ${ }^{3}$ University of Texas Medical Branch - Galveston, Department of Neurology \\ ${ }^{4}$ University of South Florida Morsani College of Medicine, Department of Neurology \\ ${ }^{5}$ Yale University, Department of Neurology
}

Mentor: Ezequiel Piccione

Program: Neurology

Type: Review/Meta-analysis

Background: In January 2020, the first case of Guillain Barre Syndrome (GBS) due to COVID-19 was documented in China. GBS is known to be postinfectious following several types of infections. Although causality can only be proven through large epidemiological studies, we intended to study this association by a thorough review of the literature.

Methods: We searched PubMed, EMBASE and Google scholar, and included all papers with English or Spanish full text and original data of patients with GBS and recent
COVID infection. Variables of interest were demographics, diagnostic investigations and the latency between arboviral and neurological symptoms. Further variables were pooled to identify GBS clinical and electrophysiological variants, used treatments and outcome. Certainty of GBS diagnosis was verified using Brighton criteria.

Results: We identified a total of 109 GBS cases. Nighty nine cases had a confirmed COVID-19 infection with an average age of 56.07 years. The average latency period between the arboviral symptoms and neurologic manifestations for confirmed COVID-19 cases was 12.2 days. The predominant GBS clinical and electromyography variants were the classical sensorimotor GBS and acute demyelinating polyneuropathy respectively. Forty cases required intensive care, 33 cases required mechanical ventilation, and 6 cases were complicated by death.

Conclusion: Studies on COVID-19-related GBS commonly reported sensorimotor demyelinating GBS with frequent facial palsy. The time between the onset of infectious and neurological symptoms suggests a post-infectious mechanism. Early diagnosis of GBS in COVID-19 patients is important as it might be associated with a severe disease course requiring intensive care and mechanical ventilation.

https://doi.org/10.32873/unmc.dc.gmerj.3.1.041

\title{
Cellular Dermatofibromas: Getting It Right the First Time Tyler D. Evans ${ }^{1}$, Erica B. Lee ${ }^{1}$, Adam V. Sutton ${ }^{1}$
}

${ }^{1}$ University of Nebraska Medical Center, College of Medicine, Department of Dermatology

\section{Mentor: Adam V. Sutton}

Program: Dermatology

Type: Review/Meta-analysis

Background: A dermatofibroma (DF) is a common, benign, skin lesion that accounts for approximately $3 \%$ of all skin specimens reviewed by dermatopathologists. A cellular dermatofibroma (CDF) is an aggressive variant that accounts for $5 \%$ of all cutaneous dermatofibromas. Given there are no established guidelines for CDF treatment, this review was aimed to review current treatment patterns in comparison to how we treat these lesions at our institution.

Methods: A PubMed literature search was performed using keyword 'cellular dermatofibroma.'

Results: There is no consensus on definitive treatments of CDF's. Clinically, it is similar to DF, but in many cases measures greater than $2 \mathrm{~cm}$ (DF is typically $<1 \mathrm{~cm}$ ). Though
CDF's have traditionally been thought to be of low malignant potential, cases of distant metastasis have been reported in the literature. There is a wide range of local recurrence rates after initial excision from 10\%-50\%. It's critically important to differentiate from dermatofibrosarcoma protuberans, a dermal neoplasm with intermediate malignant potential. Immunohistochemical (IHC) staining is the most beneficial in making this distinction. The spindle cells of CDF stain positive for factor XIIIa with CD34 staining the surrounding stroma and few cells around 